\title{
EXPLICIT LINEAR REGRESSIVE MODEL STRUCTURES FOR ESTIMATION, PREDICTION AND EXPERIMENTAL DESIGN IN COMPARTMENTAL DIFFUSIVE SYSTEMS
}

\author{
Dirk Vries * Karel Keesman* Hans Zwart ${ }^{* *}$ \\ * Dept. of Agrotechnology and Food Sciences, Systems and \\ Control Group, Wageningen University, The Netherlands \\ ** Dept. of Applied Mathematics, Faculty of EEMS, University \\ of Twente, The Netherlands
}

\begin{abstract}
A linear regressive model structure and output predictor, both in algebraic form, are deduced from an LTI state space system with certain properties without the need of direct matrix inversion. On the basis of this, explicit expressions of parametric sensitivities are given. As an example, a diffusion process is approximated by a state space discrete time model with $n$ compartments in the spatial plane and is then reparametrized. The system output can then be explicitly predicted by $\hat{y}_{k}=\hat{\theta}^{T} \phi_{k-n}-\check{\gamma}_{k-n}$ as a function of $n$, the sensor position, the parameter vector $\theta$, and input-output data. This method is attractive for estimation, prediction and insight in experimental design issues, when physical knowledge is to be preserved.
\end{abstract}

Keywords: Linear estimation, linear prediction, distributed parameter systems, regression analysis, sensitivity analysis

\section{INTRODUCTION}

Identification is about matching selected models to observations and is typically restricted to input-output data in finite time. The dynamic input-output relationships in LTI systems can be captured to get a reliable model by frequency or time domain methods which are widely available. Indeed, it is possible to estimate a (parametric) transfer function model with appropriate model order without using prior system's knowledge. However, it is not immediately clear how the structure of the transfer function model is linked to the underlying physical and/or (bio)chemical processes. Contrary to this so-called black box modelling approach, grey or white box modelling provides a model structure that is more suited for physical interpretation. Grey or white models may also aid in the derivation and/or explanation of (optimal) control solutions and experimental design issues, e.g. sensor and/or actuator placement. Therefore we would like to be able to es- timate model parameters and states while preserving the (original) physical model structure.

However, physical relevant parameters often appear non-linearly in an input-output model structure. Consequently, in general non-linear optimization solvers with (costly) iterative procedures are used, where it frequently occurs that the parameter search gets stuck in local minima. The problem gets particularly more complex when dealing with infinite dimensional systems. In that case, it is a common approach to determine a minimal basis in order to solve the estimation problem in a finite-dimensional space. See e.g: Galerkin approximation schemes (Banks and $\mathrm{Ku}-$ nisch, 1989; Baumeister et al., 1997), rational approximations (Pintelon et al., 1998), collocation methods (Söderström and Bhikkaji, 2000), minimal finite element approximations (Coca and Billings, 2002) and subspace identification techniques (Overschee, 1995). Interestingly, the latter technique has the advantage 
over classical prediction error techniques by the absence of non-linear parametric optimizations.

Our approach is to handle the parameter estimation and prediction problem, initially for an LTI infinitedimensional system, via discretization and a linear regressive parametric realization of the system in order to obtain unique estimates. However, unlike a 'data based' approach such as subspace identification, we will conserve the physical compartmental model structure due to the reasons mentioned before.

In particular, we will consider a set of finite LTI state space systems which can be regarded as compartmental diffusive systems. By the properties of such a system (denoted as $\Sigma$ ), we are able to find another realization of $\Sigma$ which is suited for linear regressive estimation and prediction. After some integral transform of $\Sigma$, we obtain a set of linear equations of the form $S^{T} M R=b$. It will be shown, that the inverse of $M$ is the resolvent of the system matrix $A$ in $\Sigma$. In the specific case that $A$ is a symmetric tridiagonal matrix, explicit solutions for the inverse of $M$ are known, see e.g. (Hu and O'Connell, 1996; Huang and McColl, 1997). The key here is to find $M^{-1}$, such that we may rewrite this as a linear regressive set of equations: $\theta^{T} \phi=\gamma$, with $\theta=\xi(\vartheta)$ a known reparametrization function of the physical parameter $\vartheta$. From hereon, it is rather straightforward to arrive at an estimate $\hat{\theta}$ using existing estimation techniques. By a simple rearrangement of terms we get an explicit expression for the output at time instant $k$, i.e. $\hat{y}\left(k \mid \hat{\theta} ; Z^{-}\right)$with $Z^{-}$the current available input-output data set.

The key objective of the paper is twofold: to show (i) the derivation and (ii) an application of explicit model structures in estimation and sensitivity analysis of diffusive-like systems, while conserving the physical model structure.

For the exact modelling case, we illustrate in section 2: (i) the methodology to arrive at an exact linear regressive representation suitable for estimation and prediction of systems characterized by the model structure $\Sigma$. Further, it will be shown how to achieve this without the use of explicit matrix inversions. In section 3 we work out (ii) by the derivation of an explicit predictor for an infinite dimensional diffusion process with boundary control at one side and a Dirichlet boundary condition at the other side, which typically can be approximated by a finite LTI model $\Sigma$. Further, sensitivity analysis of $\Sigma$ is performed. Finally, conclusions are drawn in section 4 .

\section{REALIZATION OF A LINEAR REGRESSIVE PARAMETRIC SYSTEM REPRESENTATION}

\subsection{Problem statement}

Let us start by describing the problem in some more detail. In this contribution we study the deduction of a physically-based linear regressive output predictor $\hat{y}\left(k \mid \hat{\theta} ; Z^{-}\right)$for LTI systems having a typical and known system description $\Sigma$.

Let us assume that we can rewrite the input-output system $\Sigma: y=g\left(\theta, Z^{-}\right)$described by the mapping $g$, to a mapping $f$ which is governed by a linear regressive system $\theta^{T} \phi(Z)=\gamma(Z)$ and a mapping $\tilde{f}: Z^{-} \longmapsto Y^{+}$(the predictor), with $Y^{+} \subset Y$. The motivation for finding such a system lies in the wellknown optimal properties of linear regressions, that is, unique solutions $\theta^{\star}$ can be found. Hence the problem is how to find the mapping $f$.

Summarizing, starting from the state space description,

$$
\Sigma(A, B, C, D):\left\{\begin{array}{l}
\dot{x}=A(\vartheta) x+B(\vartheta) u \\
y=C(\vartheta) x+D(\vartheta) u
\end{array}\right.
$$

with $G(\vartheta, s)=C(s I-A)^{-1} B$, we would like to find the reparametrization function $\theta=\xi(\vartheta)$ and the transformation of $\Sigma$ to the linear regressive system,

$$
\tilde{\Sigma}(\phi, \gamma):\left\{\begin{array}{l}
\theta^{T} \phi(Z)=\gamma(Z) \\
\hat{y}=\tilde{g}\left(\theta, Z^{-}\right)
\end{array}\right.
$$

\subsection{Discretization and shift-operator calculus}

In the sequel, we will consider an LTI infinitedimensional system with control at the boundary. Without loss of generality, we will focus on SISO type systems; extension to MIMO is straightforward. To simplify our analysis we consider a one-dimensional spatial domain.

The first step towards a linear regressive realization of our continuous-time PDE model is to approximate it by a finite dimensional model. We assume that we can obtain the following typical discrete-time linear state space representation $\Sigma_{d}$.

Assumption 1. There exists a discrete-time linear state space approximation of our LTI infinite dimensional system,

$$
\Sigma_{d}: \begin{cases}w^{d}(k+1) & =A(\vartheta) w^{d}(k)+B(\vartheta) u(k) \\ y(k) & =C w^{d}(k)\end{cases}
$$

and $A(\vartheta)$ and $B(\vartheta)$ linear in $\vartheta$ as

$$
\begin{aligned}
& A(\vartheta)=\bar{A}+\tilde{A} \vartheta \\
& B(\vartheta)=\bar{B}+\tilde{B} \vartheta
\end{aligned}
$$

where $w^{d} \in \mathbb{R}^{n}$ and $\vartheta$ chosen as a lumped combination of original parameters such that (3)-(5) holds.

When dealing with a diffusion process, the original 'white' parameters are the diffusion constant, the time and spatial discretization constant. Let us further introduce the forward-shift operator $q$ for manipulating the linear difference equations of (3) with 
constant coefficients (see e.g Aström and Wittenmark (1990)). Then, for what will follow in the reparametrization step, consider the transfer function $G(\vartheta, q)=C(q I-A(\vartheta))^{-1} B(\vartheta)$ as a rational function of polynomials in $q$ and $\vartheta$. For ease of notation, we will omit the arguments of $A$ and $B$ and write the time instant $k \in\{1,2, \cdots N\}$ as index.

\subsection{Linear regression by reparametrization}

Splitting the rational transfer function $G$ in a numerator $N$ and denominator part $D$, gives,

$$
N(\vartheta, q) u_{k}=D(\vartheta, q) y_{k}
$$

with $N$ and $D$ polynomials in $\vartheta$ and $q$. For instance, Pintelon et al. (1998), split $N$ and $D$ in functions of the polynomial variable $q$ (or the Laplace variable $s$ ) and the parameter vector $\theta$, so that $N(\vartheta) n(q) u_{k}=$ $B(\vartheta) d(q) y_{k}$ is considered. It is common to treat the entries of the vectors $N(\vartheta)$ and $D(\vartheta)$ as black-box parameters for further estimation and prediction. As a consequence, the link to the underlying 'white' parameters $\vartheta$ will soon be lost as the polynomial order of $N$ and/or $D$ increases. However, we will try to prevent this loss by decomposing $N($.$) and D($. not only in a shift operator dependent, but also in a (physical) parameter dependent part so to obtain polynomial coefficient matrices $\tilde{N}$ and $\tilde{D}$, that is,

$$
N=\varphi^{T} \tilde{N} \psi(q), \quad D=\varphi^{T} \tilde{D} \psi(q)
$$

with,

$$
\varphi^{T}=\left(\begin{array}{ll}
\theta^{T} & 1
\end{array}\right), \quad \psi(q)=\left(\begin{array}{llll}
1 & q & \cdots & q^{n-1} \\
q^{n}
\end{array}\right)^{T}
$$

where $\theta_{i}=\xi_{i}(\vartheta)$ for $i \in\{1,2, \cdots, n\}$ and $\xi_{i}$ a polynomial function in $\vartheta$. The final step in the linear regressive reparametrization is to rewrite (6) to the form $\theta^{T} \phi_{k}=\gamma_{k}$. Define therefore the polynomial coefficient matrices,

$$
\tilde{N}=\left(\begin{array}{cc}
\left(\bar{n}_{i j}\right) & a_{N} \\
b_{N} & c_{N}
\end{array}\right) \quad \tilde{D}=\left(\begin{array}{cc}
\left(\bar{d}_{i j}\right) & a_{D} \\
b_{D} & c_{D}
\end{array}\right)
$$

with $i, j \in\{1,2, \cdots, n\}, a . \in \mathbb{R}^{n \times 1}, b . \in \mathbb{R}^{1 \times n}$ and $c . \in \mathbb{R}$.

Furthermore, define

$$
Z_{k}=\left(U_{k} Y_{k}\right)^{T}=\left(\begin{array}{lll}
\bar{U} & u_{k+n} \mid \bar{Y} & y_{k+n}
\end{array}\right)^{T}
$$

where,

$$
\begin{aligned}
\bar{U}_{k} & =\left(\begin{array}{ll}
u_{k} & u_{k+1} \\
\cdots & u_{k+n-1}
\end{array}\right)^{T} \\
\bar{Y}_{k} & =\left(\begin{array}{lll}
y_{k} & y_{k+1} \cdots y_{k+n-1}
\end{array}\right)^{T}
\end{aligned}
$$

Substitute (7), (8), (9) and (10) in (6), a transfer function representation of $\Sigma_{d}$, and rewrite to,

$$
\varphi^{T}(\tilde{N} \mid-\tilde{D}) Z_{k}=0
$$

Now define,

$$
\begin{aligned}
& \phi_{k}=\left(\left(\bar{n}_{i j}\right) a_{N}\left(-\bar{d}_{i j}\right)-a_{D}\right) Z_{k} \\
& \gamma_{k}=\left(-b_{N}-c_{N} b_{D} c_{D}\right) Z_{k}
\end{aligned}
$$

Observe that we can rewrite (11) to a linear regressive prediction, using the definitions (12) and (13):

$$
y_{k+n}=\theta^{T} \phi_{k}-\check{\gamma}_{k}
$$

where,

$$
\check{\gamma}_{k}=c_{D}^{-1}\left(-b_{N}-c_{N} b_{D}\right) \check{Z}_{k}
$$

with $\check{Z}_{k}=\left(\begin{array}{lll}\bar{U} & u_{k+n} & \bar{Y}\end{array}\right)^{T}$. After multiplication of (11) by $q^{-n}$ (i.e. a backward time shift is applied), we may write (12)-(15) as our wanted linear regressive system:

$$
\tilde{\Sigma}_{d}: \begin{cases}\theta^{T} \phi_{k-n} & =\gamma_{k-n} \\ y_{k} & =\theta^{T} \phi_{k-n}-\check{\gamma}_{k-n}\end{cases}
$$

Notice that $g$ in (2) has become a linear function in $\theta$ and $\check{Z}_{k-n}$, see (12) and (15). This leads to the following proposition.

Proposition 1. Given system $\Sigma_{d}$ as in (3). Then,

(i) exact explicit expressions of $N(\vartheta, q)$ and $D(\vartheta, q)$ as a function of $n$ exist.

(ii) $\Sigma_{d}$ (3) can be written in the form of $\tilde{\Sigma}_{d}$ as in (16) with $\theta_{i}=\xi_{i}(\vartheta)$, a polynomial function.

Due to space limiting reasons, only a sketch of the proof is given. More details can be found in the technical report of Vries (2005).

PROOF. (i). Let $M=q I-A$ and the determinant of $M$ be denoted by $\mathbf{M}=\operatorname{det}(M)$. By writing $\mathbf{M}$ and adjoint of $M$ as Laplace expansions, and due to the linearity of $A(\vartheta)$ and $B(\vartheta)$ in $\vartheta$, it follows from induction that $\mathbf{M}$ and adj $M$ will be polynomials in $q$ and $\vartheta$ with maximum degree $n$ and $n-1$ respectively. Since $G=C M^{-1} B$ and $B$ linear in $\vartheta$ as in (5), the rest of the proof follows from decompositions of $N$ and $D$ as in (7)-(8).

(ii). Given $G(\vartheta, q)=\frac{N(\vartheta, q)}{D(\vartheta, q)}$, the transfer function of $\Sigma_{d}$. Then, $D(\vartheta, q) y_{k}=N(\vartheta, q) u_{k}$ and via direct algebra and the proof of part (i) one readily obtains $\theta^{T} \phi_{k}=\gamma_{k}$ with $\theta_{i}=\xi_{i}(\vartheta)$, a polynomial function. Notice that $\gamma_{k}$ contains $y_{k+n}$ and we can write the equivalent form $\tilde{\Sigma}_{d}(16)$ after multiplication with $q^{-n}$.

Remark 2. From the proof of Proposition 1(i) it follows directly that,

(a) The polynomial degree of $N(\vartheta, q)$ in $q$ is determined by $C$ and the adjoint of $M$. Let the entry $c_{r}$ be non-zero, with $1 \leq r \leq n$. Then the polynomial in $q$ is of maximum degree $n-r$.

(b) The polynomial degree of $N(\vartheta, q)$ in $\vartheta$ is determined by $B(\vartheta), C$ and the adjoint of $M$. Recall that $B(\vartheta)$ is linear in $\vartheta$. Again, let the entry $c_{r}$ be non-zero, with $1 \leq r \leq n$. Then the maximum degree of polynomial in $\vartheta$ is $n-r+1$.

(c) $a_{N}=b_{N}=c_{N}=0$, due to the maximum degree $n-1$ of the polynomial adj $M$ in $\vartheta$ and $q$. More generally, if the maximum degree of polynomials 
is $n-r, 1 \leq r \leq n$, then the lower $r$ rows of $\tilde{N}$ is filled with zeros. This result is caused by causality of $\Sigma_{d}$.

Remark 3. (Identifiability).

After defining $\Phi=\left(\phi_{1}, \cdots, \phi_{N}\right)$ and $\Gamma=\left(\gamma_{1}, \cdots, \gamma_{N}\right)$ one obtains: $\theta^{T} \Phi=\Gamma$. Then, the least-squares estimate is given by: $\hat{\theta}=\left(\Phi \Phi^{T}\right)^{-1} \Phi \Gamma^{T}$. Consider $\tilde{N}$ a matrix originating from a polynomial of maximum degree $n$ in $\vartheta$ and maximum degree $n-1$ in $q$. Then, a unique solution is found as long as $\operatorname{ker}(\Phi) \in \emptyset$ or equivalently (i) $\operatorname{rank}(\Phi)$ is full or (ii) $\operatorname{det}\left(\Phi \Phi^{T}\right) \neq 0$. It is straightforward to show that $Z_{k} \neq 0$ is a sufficient condition for a unique solution.

Remark 4. (Predictability). Note that, in order to predict $y_{k+n}$, (i) if $c_{D}=0,\left(-b_{N}-c_{N} b_{D}\right) \check{Z}_{k}=0$ for all $t_{k}$, or, (ii) $c_{D} \neq 0$, should hold. This follows from the assumption that $y_{k+n}$ exists, see (14)-(16).

Consequently, we will not need the calculation of $M^{-1}$ by numerical matrix inversion methods, as we have an explicit series solution. A diffusion example case with Dirichlet boundary conditions will be used to further illustrate the mentioned sequence of steps.

\subsection{Sensitivity analysis}

So far we have derived the model structure representation $\gamma_{k}=\theta^{T} \phi_{k}$, with $\theta$ explicitly depending on the physical $\vartheta$. Let us now, on the basis of this, investigate its sensitivities. We start by realizing that the regression model of $\tilde{\Sigma}_{d}$ exhibits an input-output mapping from $U_{k}$ to the regression response $\gamma_{k}$. This becomes clear by substituting the convolution sum, i.e. in matrix form, $Y_{k}=H U_{k}$ in (13) with $H \in \mathbb{R}^{n_{H} \times n_{H}}$. The entries of the Hankel matrix $H$ contain the Markov parameters and can directly be found by an impulse response as a function of the real parameters as $\theta_{0}$. Consequently, for $n_{H}=n$ (notice that $n_{H}$ should be large in order to satisfactorily approximate the infinite impulse response, so that $n$ will then be limiting),

$$
\phi_{k}=\left(\left(\bar{n}_{i j}\right)-\left(\bar{d}_{i j}\right) H\right) U_{k}
$$

Further, denote the real (unknown) parameters $\theta_{0}$. We obtain the sensitivities of $\gamma$ by differentiation and substitution of (17) in (16), which gives,

$$
\begin{gathered}
\gamma_{\theta} \triangleq \frac{d \gamma}{d \theta}=\left(\left(\bar{n}_{i j}\right)-\left(\bar{d}_{i j}\right) H\right) U \\
\gamma_{\theta U} \triangleq \frac{d \phi}{d U}=\left(\bar{n}_{i j}\right)-\left(\bar{d}_{i j}\right) H
\end{gathered}
$$

An explicit solution for $\gamma_{\theta}$ and $\gamma_{\theta U}$ in a compartmental diffusion system (as in the next section) is reported in (Vries, 2005).

\section{RESULTS}

\subsection{A boundary controlled diffusion process}

Consider an infinite dimensional system $\Sigma^{e}$ of parabolic type on $[0, \infty) \times[0, \infty)$,

$$
\Sigma^{e}: \begin{cases}\frac{\partial w}{\partial t}(x, t) & =\alpha^{2} \frac{\partial^{2} w}{\partial x^{2}}(x, t) \\ w(0, t) & =u(t), w(x, 0)=w_{0}(x) \\ y(t) & =w\left(x^{*}, t\right)\end{cases}
$$

where $w_{0}(x) \in L_{2}(0, \infty), x^{*} \in[0, \infty)$ and $U=\mathbb{R}$. This system is a boundary control system, see (Curtain and Zwart, 1995).

This implies that for smooth initial condition $w_{0}$ and smooth control $u$, with $w_{0}(0)=u(0)$, there exists a unique classical solution of $\Sigma^{e}$ with $w(x, t) \in$ $L_{2}(0, \infty)$ for all $t \geq 0$. The solution to this problem when applying a step input $u(t)=1_{[0, \infty)}(t)$ is well-known and is given by the output $y(t)=$ erfc $\left(x^{*} /(2 \alpha \sqrt{t})\right) 1_{[0, \infty)}(t)$, where $\alpha^{2}$ can be interpreted as the heat transfer constant or diffusion constant. $\Sigma^{e}$ may thus describe a heating process of an infinitely thick homogeneous wall or equivalently a diffusion phenomenon of e.g. penetrating pollutant concentrations from the earth surface into deeper layers.

The unknown parameter $\alpha^{2}$ can be estimated via nonlinear estimation techniques using either an analytical or numerical solution of (20). As said, this may lead to local solutions and a high computational effort. As an alternative, we seek an approximation of (20) and denoted as $\Sigma_{d}^{e}$, such that it is equivalent to $\tilde{\Sigma}_{d}$ as in (16). In the sequel, we will follow the same name conventions for the subsections as in section 2 .

\subsection{Discretization and shift-operator calculus}

After applying central finite differences, we get the discrete-time compartmental system $\Sigma_{d}^{e}$ in the form of (3) by defining the lumped parameter,

$$
\vartheta=\alpha^{2} \Lambda
$$

where $\Lambda=\Delta_{t} / \Delta_{x}^{2}$ denotes the fraction of the time differential operator $\Delta_{t}$ and the place differential operator $\Delta_{x}$. According to (4), $\bar{A}$ becomes the identity matrix $I, I \in \mathbb{R}^{n \times n}$ and $\tilde{A}$ a symmetric 3 -banded Toeplitz matrix with on the main diagonal $\tilde{a}_{i i}=-2$ and all entries on the first sub- and superdiagonal $\tilde{a}_{i+1, i}=\tilde{a}_{i-1, i}=1$, and further, $B=\left(\begin{array}{llll}\vartheta & 0 & \cdots & 0\end{array}\right)^{T}$. The observation $y(t)=w\left(t, x^{*}\right)$ is approximated by $C w_{k}^{d}$, with $C$ mapping a 'point' observation at the $j$ th compartment (i.e $c_{j^{*}}=1, j^{*} \in j$ ). Notice that a grid with $n$ points directly leads to $n$ states, because we have started with one state variable and one spatial direction in the PDE model $\Sigma^{e}$. 
After forward-shift operations on $\Sigma_{d}^{e}$, it will later on become clear that it is convenient to introduce,

$$
M=q I-A=\left(\begin{array}{cccccc}
\nu & 1 & & & & 0 \\
1 & \nu & 1 & & & \\
& \cdot & \cdot & . & & \\
& & & 1 & \nu & 1 \\
0 & & & & 1 & \nu
\end{array}\right) \vartheta
$$

with $\nu=\left(q-\left(\bar{a}_{i i}+\tilde{a}_{i i} \vartheta\right)\right) / \vartheta=(q-1) / \vartheta+2$.

\subsection{Linear regression by reparametrization}

We can now decompose the numerator $N$ and denominator $D$ of the transfer function $G$ as in (7), given $B$ and $C$. We obtain the following,

Proposition 5. Given $\Sigma_{d}^{e}(A, B, C)$, with $A, B$ and $C$ as defined above, then an exact explicit expression for $\tilde{\Sigma}_{d}^{e}(\phi, \gamma, g)$ is given by a series solution.

PROOF. Following Hu and O'Connell (1996), we can write $M^{-1}$ as a quotient of series solutions in $\nu$. The rest of the proof is analogous to the proof of Proposition 1 .

Because we are dealing with a single parameter, the polynomial functions $\xi_{i}$ become $\xi_{i}(\vartheta)=\vartheta^{n-i+1}, i=$ $1,2, \cdots, n$, so that $\theta^{T}=\left(\vartheta^{n} \vartheta^{n-1} \cdots \vartheta\right)$.

Furthermore, equation (9) becomes indeed,

$$
\tilde{N}=\left(\begin{array}{cc}
\left(\bar{n}_{i j}\right) & 0 \\
0 & 0
\end{array}\right), \quad \tilde{D}=\left(\begin{array}{cc}
\left(\bar{d}_{i j}\right) & 0 \\
b_{D} & c_{D}
\end{array}\right)
$$

It should be noted that $(\bar{n})$ and $(\bar{d})$ become lower triangular matrices with properties as stated in Remark 2 . Now, denote for notational reasons $n^{*}=n-j^{*}$. We find by direct algebra an explicit expression for (22) and obtain the following.

Proposition 6. Given the SISO-LTI system (20), then the entries in (22) are given by

$$
\begin{gathered}
\tilde{d}_{i j}= \begin{cases}(-1)^{i+j-2}\left(\begin{array}{c}
n+i \\
n-i+1
\end{array}\right)\left(\begin{array}{l}
i-1 \\
j-1
\end{array}\right) & \text { if } i \geq j \\
0 & \text { elsewhere }\end{cases} \\
\tilde{n}_{i j}= \begin{cases}(-1)^{i+j-2}\left(\begin{array}{c}
n^{*}+i \\
n^{*}-i+1
\end{array}\right)\left(\begin{array}{c}
i-1 \\
j-1
\end{array}\right) & \text { if } i \geq j \wedge \\
0 & i \leq n^{*}+1\end{cases} \\
\hline
\end{gathered}
$$

where $\left(\begin{array}{l}n \\ k\end{array}\right)$ denotes the binomial coefficient of $n$ over $k$.

Observe from Proposition 6 and the definition in (9), that (22) is specified with

$$
\begin{aligned}
\bar{n}_{i j} & =\bar{n}_{\{1 \cdots n-1\}\{1 \cdots n-1\}} & \bar{d}_{i j} & =\bar{d}_{\{1 \cdots n-1\}\{1 \cdots n-1\}} \\
b_{D} & =\tilde{d}_{n\{1 \cdots n-1\}} & c_{D} & =\tilde{d}_{n n}
\end{aligned}
$$

Now, we rearrange terms as in (10)-(13) in order to get the regression vector $\phi_{k}$ and the so called response vector $\gamma$. Each row $i$ of the regression matrix $\Phi=\left[\phi_{1} \cdots \phi_{k}\right]$, with column entry $k \in$ $\{1,2, \cdots, N\}$, becomes,

$$
\Phi_{i, k}=\sum_{j=1}^{i}\left(\bar{n}_{i j} u_{k+j-1}-\bar{d}_{i j} y_{k+j-1}\right)
$$

and each entry $k$ of the so-called response vector $\gamma$ (notice that here $i \in\{1, \cdots, n+1\}$ ), is given by,

$$
\gamma_{k}=(-1)^{n} \sum_{i=1}^{n+1}(-1)^{i-1}\left(\begin{array}{c}
n \\
i-1
\end{array}\right) y_{k+i-1}
$$

From (14) it follows that a predictor of the output $\hat{y}_{k+n}$, based on the uniquely estimated parameters $\hat{\theta}$ is equivalently written as,

$$
\begin{aligned}
\hat{y}_{k+n} & =\hat{\theta}^{T} \Phi_{i, k}-\check{\gamma}, \quad \text { with, } \\
\check{\gamma} & =(-1)^{n} \sum_{i=1}^{n}(-1)^{i-1}\left(\begin{array}{c}
n \\
i-1
\end{array}\right) y_{k+i-1}
\end{aligned}
$$

Observe that $\tilde{\Sigma}_{d}^{e}$ has only become dependent on the number of compartments $n$, the sensor position $j^{*}$, the parameters $\theta$, and available input-output data.

\subsection{Sensitivity Analysis}

Evaluation of the sensitivity matrix $\gamma_{\theta U}$ gives us for a given impulse response as a function of $\theta_{0}$ valuable information about sensitivities, independent of $\theta$ or $U$. For the diffusion system $\Sigma_{d}^{e}$ with $\alpha=0.4$, $n=n_{H}=8$, we calculated $\gamma_{\theta U}$ at different sensor positions. Although seeming counter-intuitively, figure 1 shows that it is recommended to put the sensor at the first compartment which is closest to the boundary input, see also (Keesman and Stigter, 2002). In this compartment, all sensitivities are excited, whereas the magnitude rapidly diminishes if the sensor is placed further away. The eigenvalues in the right graph in figure 2 confirm this finding, but also show that there is a risk of ill-conditioning of $\gamma_{\theta U}$. The left graph in figure 2 show the parametric sensitivity $\gamma_{\theta}$ when applying a step input $1_{k}, k=1, \cdots, n$, which is obtained by summation of the columns of $\gamma_{\theta U}$. Again, it seems favorable to place a sensor in the first compartment. Interestingly, we notice that a constant input may not be exciting enough if we compare it with the impulse response (cf. figure 1). This indicates that a pulsating sequence with certain switching probability is to be preferred.

\section{CONCLUDING REMARKS}

With the proposed procedure it is possible to conserve the underlying compartmental physical model structure in combination with linear regressive parameter estimation. The transformation to a newly obtained 
Fig. 1. Contour plots of the sensitivity matrix $\gamma_{\theta U}$ at different sensor positions $j^{*}$
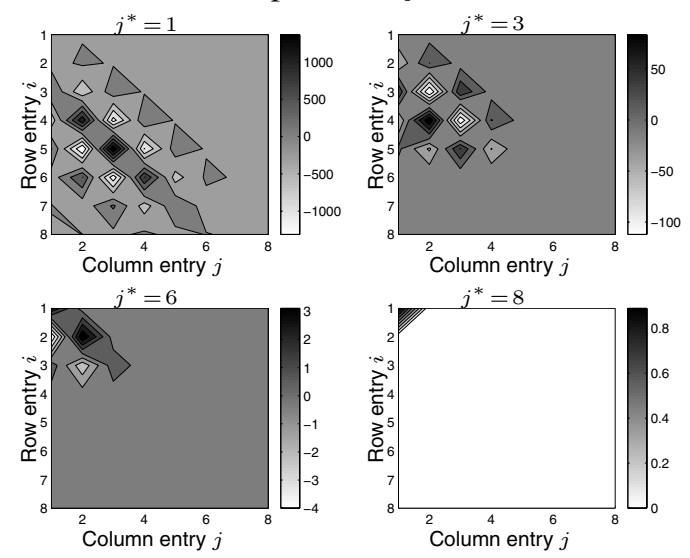

Fig. 2. Parametric sensitivities $\gamma_{\theta}$ for a step input and eigenvalues of the sensitivity matrix $\gamma_{\theta U}$, the $(\times),(\circ),(*)$ and $(\triangle)$-marked lines correspond to a sensor at $j^{*}=1,3,6$ and 8 respectively.
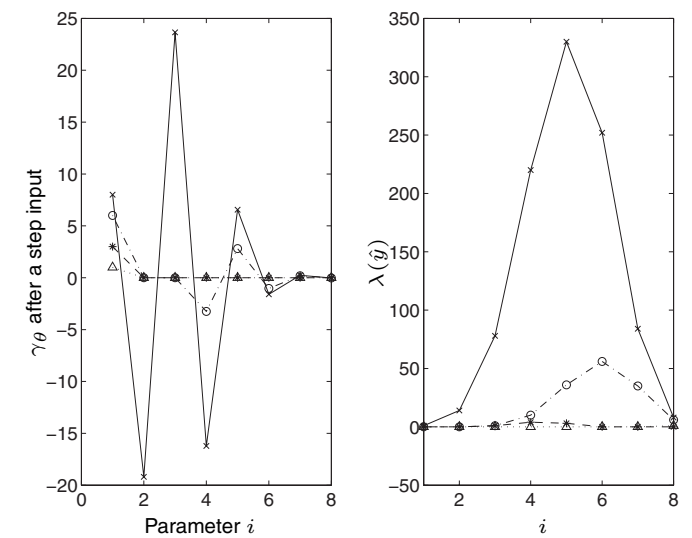

linear regressive system $\tilde{\Sigma}$ from a state space system $\Sigma$ is based on linearity of the system and linearity in the parameter $\vartheta$ of $A(\vartheta)$ and $B(\vartheta)$. An a priori analysis can be made on $\phi$ to inspect the (parametric) sensitivities.

In practice however, there will be measurement noise deteriorating both the inputs and outputs of the linear regressive system $\tilde{\Sigma}_{d}$ in a correlated manner. This unwanted effect can be tackled by e.g. TLS techniques or prefiltering and is subject to further study.

The sketched methodology is attractive for estimation, prediction and insight in experimental design issues, when physical knowledge in the model structure is to be preserved. Although the derivation of the structures can be quite elaborative, they may aid in the insight of the model structure properties. For compartmental diffusive models we have derived these linear regressive structures.

\section{ACKNOWLEDGMENTS}

This work was supported by the Technology foundation STW under the project number WWI.6345.

\section{REFERENCES}

Aström, K.J. and B. Wittenmark (1990). Computercontrolled Systems: Theory and Design. Prentice Hall, NJ.

Banks, H.T. and K. Kunisch (1989). Estimation Techniques for Distributed Parameter Systems. Birkhäuser, Boston.

Baumeister, J., W. Scondo, M.A. Demetriou and I.G. Rosen (1997). On line parameter estimation for infinite-dimensional dynamical systems. SIAM J. Control Optim. 35:2, 678-713.

Coca, D. and S.A. Billings (2002). Identification of finite dimensional models of infinite dimensional dynamical systems. Automatica 38, 1851-1865.

Curtain, R.F. and H.J. Zwart (1995). An Introduction to Infinite Dimensional Linear Systems Theory. Springer-Verlag, New York.

Hu, G.Y. and R.F. O'Connell (1996). Analytical inversion of symmetric tridiagonal matrices. J. Phys. A: Math. Gen. 29, 1511-1513.

Huang, Y. and W.F. McColl (1997). Analytical inversion of general tridiagonal matrices. J. Phys. A: Math. Gen. 30, 7919-7933.

Keesman, K.J. and J.D. Stigter (2002). On compartmental modelling of mixing phenomena. In: 15th IFAC World Congress on Automatic Control. (CD-Rom). Barcelona, Spain.

Overschee, P. Van (1995). Choice of state-space basis in combined deterministic-stochastic subspace identification. Automatica 31:12, 1877-1883.

Pintelon, R., P. Guillaume, G. Vandersteen and Y. Rolain (1998). Analysis, development and applications of tls algorithms in frequency domain system identification. SIAM J. Matrix Anal. Appl. 19:4, 983-1004.

Söderström, T. and B. Bhikkaji (2000). Reduced order models for diffusion systems via collacation methods. In: IFAC 12th Symposium on System Identification. (CD-Rom). Santa Barbara, California.

Vries, D. (2005). Explicit expressions for estimation, prediction and parametric sensitivities in a compartmental diffusion model: a linear regression approach. Technical Report WWI6345-WU05081. Wageningen University. 